Relations industrielles

Industrial Relations

\title{
L’avenir du syndicalisme dans la fonction publique fédérale du Canada
}

\section{The Future of Public Service Unionism in Canada}

\section{Claude A. Edwards}

Volume 29, numéro 4, 1974

URI : https://id.erudit.org/iderudit/028556ar

DOI : https://doi.org/10.7202/028556ar

Aller au sommaire du numéro

\section{Éditeur(s)}

Département des relations industrielles de l'Université Laval

\section{ISSN}

0034-379X (imprimé)

1703-8138 (numérique)

Découvrir la revue

\section{Citer cet article}

Edwards, C. A. (1974). L'avenir du syndicalisme dans la fonction publique fédérale du Canada. Relations industrielles / Industrial Relations, 29(4), 804-824. https://doi.org/10.7202/028556ar
Résumé de l'article

L'auteur fait le point sur l'état actuel de la négociation collective dans la fonction publique fédérale canadienne. Il souligne que l'habitude de donner des augmentations salariales en termes procentuels plutôt qu'en termes absolus a considérablement augmenté les écarts de traitement au détriment des fonctionnaires à faibles revenus et que ceci va créer d'importantes tensions dans les futures négociations. Il discute également de la consultation institutionnalisée de la négociation concertée et du rôle que sera appelée à jouer la Commission de la fonction publique.
Tous droits réservés @ Département des relations industrielles de l'Université Laval, 1974
Ce document est protégé par la loi sur le droit d'auteur. L'utilisation des services d'Érudit (y compris la reproduction) est assujettie à sa politique d'utilisation que vous pouvez consulter en ligne.

https://apropos.erudit.org/fr/usagers/politique-dutilisation/ 


\title{
L'avenir du syndicalisme dans la fonction publique fédérale du Canada
}

\author{
Claude A. Edwards
}

L'auteur fait le point sur l'état actuel de la négociation collective dans la fonction publique fédérale canadienne. Il souligne que l'habitude de donner des augmentations salariales en termes procentuels plutôt qu'en termes absolus a considérablement augmenté les écarts de traitement au détriment des fonctionnaires à faibles revenus et que ceci va créer d'importantes tensions dans les futures négociations. Il discute également de la consultation institutionnalisée de la négociation concertée et du rôle que sera appelée à jouer la Commission de la fonction publique.

\section{LES PREMIÈRES NÉGOCIATIONS DANS LA FONCTION PUBLIQUE FÉDÉRALE}

Il ne saurait être question de parler en termes d'avenir sans d'abord examiner brièvement le passé et le présent puisque toute prédiction quant à ce que nous réserve l'avenir sera fondée sur ce que nous a valu le passé et sur ce que nous vaut le présent.

Il y a à peine six ans qu'a été instituée la négociation collective dans la Fonction publique fédérale. La première ronde s'est déroulée dans un climat d'euphorie qu'avait caractérisé la bonne foi manifeste des deux parties, par un recours restreint de la part des deux parties à des experts aux séances de négociation et par le nombre de difficultés presque insurmontables. La tâche de transposer l'ancien régime de classification en 72 unités de négociation en fonction d'un système axé

EDWARDS, C.A., président, Alliance de la fonction publique du Canada, Ottawa.

* Communication présentée au Congrès de l'Institut canadien de recherche en relations industrielles, Toronto, 1974.

Paper presented at the Canadian Industrial Relations Research Institute annual meeting, Toronto, 1974. 
sur les occupations s'est révélée d'une ampleur monumentale. Nous avions réussi à faire intégrer à ces conventions collectives bien des améliorations au nom de nos membres et plus encore au nom de ces employés qu'on désignait précédemment sous le nom d'employés à taux régnants.

Le Conseil du Trésor, et tout me porte à le croire, a certes jugé que les premiers négociateurs ministériels avaient fait cadeau de tout le bazar au cours de la première ronde. Nous avons vu de nouveaux visages se présenter aux séances de négociation et nous avons éprouvé beaucoup plus de difficulté à obtenir des améliorations au cours de la deuxième ronde; c'en était fait de la lune de miel et cependant, les employés, peu rompus aux rouages de la négociation collective, s'imaginaient vraisemblablement qu'il suffisait à leurs représentants de revendiquer pour que le Conseil du Trésor accède à leurs propres demandes. Et si la convention collective ne reflétait pas leurs propres aspirations, c'était, de toute évidence, parce que leurs négociateurs n'avaient tout simplement pas demandé ou revendiqué.

\section{LA SITUATION ACTUELLE}

La présente ronde de négociation a été encore plus rude et, par la même occasion, les besoins et les attentes des employés n'ont cessé de croître. On peut attribuer cette intensification de leurs espérances à divers facteurs dont le principal, en ce moment, est sans contredit la montée en flèche de l'Indice des prix à la consommation. L'inflation met à rude épreuve l'employé et plus encore celui qui exerce au palier inférieur de l'échelle de rémunération. De plus, le petit écran a envahi la salle de séjour du pauvre pour faire miroiter à ses yeux le train de vie du riche alors que le pauvre, loin de pouvoir s'offrir ce train de vie, a grand peine à joindre les deux bouts. Le prix des denrées alimentaires essentielles : viande, lait, etc., augmente de semaine en semaine et non plus de mois en mois ou d'année en année comme autrefois. Les loyers ne cessent d'augmenter. Son rêve de devenir propriétaire s'est dissipé en fumée et, dans ce contexte, il se sent de plus en plus frustré. On verra donc les agneaux se transformer en lions. Ils feront table rase de l'autorité et des règlements. Ils ne veulent plus se conformer aux règles du jeu. Ils s'attendent que leurs revendications soient suivies de mesures immédiates, soutenues et efficaces. C'est avec sarcasme qu'ils rejetteront comme de peu d'importance sinon aucune tout gain qu'en temps normal ils auraient pu juger fort considérable. 
Les fonctionnaires voient les employés de l'industrie privée refuser des augmentations annuelles de 12 p. 100, des augmentations horaires de $\$ 2.90$ dans les métiers de la construction et ne manquent pas d'établir des comparaisons salariales avec leur propre convention collective. Il est donc fort peu probable qu'ils ratifient les projets de conventions collectives négociées au moyen de comparaisons empiriques et traditionnelles avec la rémunération accordée aux employés qui s'acquittent des mêmes tâches dans le secteur privé. Les membres ont rejeté, jusqu'à présent cette année, cinq accords provisoires alors qu'ils n'en avaient rejeté qu'un seul au cours des années précédentes. Un des projets de convention rejetés pourvoyait à des augmentations salariales de plus de 30 p. 100, étalées sur $25 \frac{1}{2} 2$ mois, ainsi qu'à d'autres avantages sociaux. L'accord provisoire était intervenu suite aux pressions d'une grève illégale et les employés auraient obtenu par voie de négociation, une augmentation salariale sans précédent dans l'histoire de la Fonction publique. Les membres n'ayant pas ratifié cet accord provisoire, le différend sera accommodé éventuellement par décision arbitrale exécutoire.

Il serait toutefois injuste et on serait fort mal avisé d'attribuer le malaise actuel uniquement aux facteurs du milieu immédiat. Voilà bien plus d'une décennie que cette tension ne cesse d'augmenter et qu'elle n'est que l'aboutissement de cette situation de plus en plus caractérisée qu'occupent les travailleurs au bas de l'échelle,ceux-là mêmes qui ne bénéficient aucunement de l'essor et de la prospérité du Canada.

L'amélioration de la condition relative des travailleurs canadiens qui n'ont pas encore obtenu leur juste part des fruits de l'essor économique et de la mise en valeur du pays s'inscrit parmi les plus grandes difficultés que la négociation collective, tant dans le secteur public que dans le secteur privé, sera appelée à résoudre.

Il se trouve encore, dans le secteur privé, des travailleurs non-syndiqués qui touchent à peine et rarement plus que le salaire minimum garanti par la loi et qui, dans certains cas, ne touchent même pas le taux qu'est censée leur garantir la loi. Il est regrettable de constater, à la lumière des réalisations par ailleurs remarquables du syndicalisme canadien, que près d'un siècle après la fondation des premiers syndicats au pays et trente-cinq ans après que la loi eût mis les employés à couvert des méthodes coercitives des employeurs, afin de garantir l'autonomie des syndicats, plus des deux tiers des travailleurs canadiens ne sont pas encore constitués en syndicats. 


\section{LES CAUSES DU MÉCONTENTEMENT}

Cette dernière décennie aura été caractérisée par de remarquables progrès au sein des institutions municipales et provinciales et même là, on pourrait fort bien améliorer et la forme et le fond de la négociation collective de plusieurs fonctions publiques provinciales. La difficulté que sont appelés à résoudre les syndicats de la Fonction publique fédérale ne consiste pas à donner plus d'ampleur à leurs effectifs qui se situent à quelques dixièmes pour cent près du total ni à obtenir le droit de négocier - droit qu'ils ont obtenu en 1967 - mais bel et bien à obtenir un revenu plus considérable pour ceux de leurs membres qui, au bas de l'échelle, doivent lutter d'arrache-pied pour survivre. Il ne fait aucun doute que l'inflation effrénée nous aura sensibilisés davantage aux tribulations des membres les moins bien rémunérés ainsi qu'aux difficultés qu'ils éprouvent à joindre les deux bouts à même un revenu qui se situe au seuil de la pauvreté. Il se pourrait que notre souci d'obtenir aux fonctionnaires une rémunération comparable à la rémunération consentie dans le secteur privé nous ait fait consentir, dans le cas des petits salariés, à des accords salariaux qui, bien que statistiquement justifiables n'en étaient pas moins moralement révoltants. Le syndrome de «l'augmentation procentuelle $\gg$ qui, depuis une dizaine d'années, s'est propagé sur le plan de la négociation collective au Canada, s'est soldé par des augmentations salariales censées maintenir un juste rapport entre les travailleurs aux divers niveaux de revenus mais qui se résument effectivement à un camouflage statistique de la disproportion croissante entre l'opulence relative des employés aux niveaux supérieurs de rémunération et l'indigence relative de ceux qui exercent à petit salaire. Les augmentations salariales procentuelles ne correspondent pas à l'accroissement ou au manque d'accroissement du pouvoir d'achat de ceux qui en bénéficient, comme pourrait fort bien le démontrer celui qui ne touche aucun revenu. Il ne saurait être question d'augmenter le pouvoir d'achat de celui qui ne touche aucun revenu en lui consentant une augmentation de 10 p. 100.

Examinons, à titre d'exemple, la condition de trois salariés qui, au cours des années '60, gagnaient respectivement $\$ 3,000, \$ 18,000$ et $\$ 50,000$ et qui ont bénéficié par la suite d'augmentations annuelles de 5 p. 100. En supposant que le travailleur le moins bien rémunéré n'ait pas eu à payer d'impôt sur le revenu, que le travailleur au traitement moyen ait dû acquitter un impôt de 30 p. 100 et le mieux rémunéré, un impôt de 40 p. 100 , l'augmentation annuelle du pouvoir d'achat, au terme de la décennie, serait de $\$ 1,654$ dans le cas du moins bien rému- 
néré, de $\$ 6,947$ pour le salarié moyen et de $\$ 16,540$ pour l'employé le mieux rémunéré.

Le pouvoir d'achat de l'employé le mieux rémunéré dépassait de $\$ 27,000$, en 1960 , celui de l'employé le moins rémunéré. En 1969, la différence du pouvoir d'achat était de $\$ 41,886$ entre l'employé le mieux rémunéré et l'employé le moins rémunéré.

L'augmentation globale du pouvoir d'achat, au cours de la décennie, s'établissait donc à $\$ 37,734$ dans le cas de l'employé le moins rémunéré, à $\$ 158,482$, pour l'employé à salaire moyen et à $\$ 377,337$, pour l'employé le mieux rémunéré.

Dans le contexte nord-américain, la plupart des travailleurs, dont les membres de l'Alliance de la Fonction publique, ne croient pas qu'on doive rémunérer également tous les travailleurs. Ils estiment qu'il doit régner, en matière de salaires et de traitements, un écart convenable propre à refléter les divers apports des employés et à les encourager à perfectionner leurs compétences et leur éducation et à se charger de plus lourdes responsabilités. Mais je ne crois pas qu'ils approuvent cet écart de plus en plus marqué entre les travailleurs au bas de l'échelle et ceux qui en occupent les plus hauts échelons ni qu'ils souscrivent à ce partage régressif du revenu national, corollaire inévitable de notre présent concept salarial.

Il n'existe pas, à mon avis, de problème plus critique que soit appelée à résoudre notre société contemporaine. La situation actuelle se résume à ceci : ceux qui font déjà bonne chère feront, le temps aidant, bombance alors que ceux qui ont à peine de quoi se mettre sous la dent devront bientôt se contenter de subsister, à condition d'y parvenir, à même les miettes. La masse des petits salariés du pays va-t-elle acquiescer paisiblement et bien longtemps encore à ce concept salarial manifestement discriminatoire? À quel point peut-elle endurer sans se révolter?

\section{LA POSITION DE L'ALLIANCE DE LA FONCTION PUBLIQUE}

L'Alliance de la Fonction publique cherche les moyens d'atténuer les pires répercussions que lui a values ce cercle salarial régressif dont elle n'a pas réussi à sortir depuis le début des négociations, en 1967. Les préoccupations que nous vaut cette situation trouvent leur écho dans quelques-uns des accords que nous avons conclus récemment et aux termes desquels on a modifié la formule classique des augmentations procentuelles en accordant un plus fort pourcentage aux plus bas niveaux, 
soit en termes de pourcentage, soit sous forme d'augmentations procentuelles intégrées à des relèvements forfaitaires. La récente augmentation générale de $\$ 500$ consentie aux employés de la Fonction publique en contrepartie de l'augmentation du coût de la vie n'est qu'un simple pas dans la bonne voie et nous essaierons d'en franchir d'autres, d'une manière ou d'une autre. Cette augmentation, en termes de pourcentages, équivaut, même avant les retenues d'impôt, à 10 p. 100, dans le cas de l'employé qui gagne $\$ 5,000$ et vaut 1 p. 100 environ à l'employé qui occupe un poste parmi les mieux rémunérés.

D'autre part, ni le Conseil du Trésor en sa qualité d'employeur, ni les syndicats avec lesquels le Conseil négocie n'ont cette latitude de renoncer de plein gré au concept salarial de la collectivité. Attendu que le gouvernement doit s'en tenir rigoureusement à une politique d'équilibre entre la rémunération qu'il accorde à ses employés et celle dont bénéficient les contribuables qui s'acquittent des mêmes tâches, les agents négociateurs n'obtiendront que très difficilement pour leurs membres des taux de rémunération supérieure à ceux que paient les bons employeurs du secteur privé. On ne saurait donc obtenir de changement appréciable qu'à la condition que les employés et les agents négociateurs, du pays tout entier, s'efforcent par tous les moyens de mettre au point des formules et des modalités de rajustement de la rémunération propres à faire échec à ce rapport salarial socialement immoral et artificiellement propagé depuis une vingtaine d'années par le truchement des augmentations procentuelles.

Quand on pense que la rémunération moyenne dans la Fonction publique fédérale se situe actuellement à quelque $\$ 9,700^{1}$ et que, selon les données les plus récentes du Conseil du Trésor, la Fonction publique compte environ 120,000 employés qui gagnent moins de $\$ 10,000$ par année, on comprend facilement que les frustrations de ces employés puissent se traduire ouvertement de bien des façons.

\section{DES NÉGOCIATIONS DIFFICILES À VENIR}

Les employés prendront aussi bien leurs syndicats que l'employeur pour cibles de leurs déceptions. Si les grèves impromptues et les manifestations risquent de se multiplier, il en sera de même des tentatives de changements d'agent négociateur ou de dirigeants syndicaux et pourtant,

1 Moyenne de 240,000 personnes, le 31 décembre 1973, à l'exception des Forces armées, des employeurs distincts et des sociétés de la Couronne. 
les autres agents ou les autres dirigeants syndicaux n'auront guère de solutions faciles à proposer aux problèmes.

Il ne sera guère facile de faire régner la discipline au sein des syndicats et on peut s'attendre à ce qu'on fasse fi des règlements du syndicat comme de ceux de l'employeur. Les rapports contractuels n'en seront que plus difficiles car ni le syndicat ni la direction n'auront la certitude que les employés se conformeront aux accords intervenus. L'employeur pourrait fort bien, dans un tel contexte, ne pas consentir ses conditions les plus avantageuses afin de compter sur certaines réserves en cas de non ratification ou de grève impromptue. Le représentant syndical, d'autre part, n'acceptera pas et ne recommandera pas volontiers une offre qu'il jugerait acceptable dans des circonstances ordinaires, sans s'être assuré d'abord du consentement majoritaire de ses commettants.

Voilà autant de facteurs qui ne peuvent que rendre la négociation plus pénible encore, donner lieu à moins d'accords négociés, à des recours plus nombreux aux rouages d'accommodement des différends et, vraisemblablement, à des affrontements plus nombreux et à une agitation plus manifeste.

Les frustrations attribuables à l'inflation et à une politique salariale qui n'a de cesse d'élargir ce fossé entre les employés moins rémunérés et les employés mieux rémunérés pourraient bien mettre à rude épreuve la faculté de tous ceux qui œuvrent sur le plan des relations du travail à perpétuer un système mis au point dans le but d'instituer et de maintenir, dans le cadre de la négociation collective, des rapports harmonieux entre l'employeur et les employés et ce, avec un minimum de tension.

\section{LA CONSULTATION}

Le système des relations de travail dans la Fonction publique a été caractérisé par un recours marqué à la consultation des parties comme moyen de résoudre des difficultés ou d'arrêter certaines conditions d'emploi. C'est ainsi que, règle générale, on a pu élaborer et arrêter les conditions de travail d'application universelle à la Fonction publique. Le carrefour de consultation le plus remarquable, pour la Fonction publique fédérale, c'est le Conseil national mixte dont ce sera, la semaine prochaine, le $30 \mathrm{e}$ anniversaire de fondation.

Il existe un besoin manifeste de rouages grâce auxquels les parties pourraient arrêter et les conditions de travail et les avantages sociaux sem- 
blables puisque les employés sont portés à comparer leurs conditions de travail à celles de leurs collègues. Les décisions prises par l'intervention $\mathrm{du}$ Conseil en ont permis l'application universelle et leur mise en vigueur à la même date, solution qu'il serait difficile à l'extrême d'obtenir dans le cadre de la procédure normale de négociation puisque les diverses conventions collectives viennent à expiration à des dates différentes. Le recours au Conseil national mixte prévient aussi, tant de la part de l'employeur que de celle des syndicats, la «négociation spéculative », situation qui se présenterait vraisemblablement si ces conditions faisaient l'objet de négociations distinctes pour chaque unité.

Il faudra, selon moi, que les syndicats comme l'employeur mettent des ressources plus considérables à la disposition du Conseil national mixte s'ils veulent que cet organisme demeure un carrefour de consultation. À l'heure actuelle, le Conseil s'acquitte principalement de ses fonctions par l'entremise de comités composés d'employés de la direction et d'employés des syndicats qui occupent tous, à titre officiel, des emplois à plein temps. On ne peut facilement réunir les comités, les décisions s'en trouvent différées, on ne peut donner suite rapidement aux modifications recommandées et les employés affectés par les décisions ont perdu patience.

M. Finkelman, dans son rapport sur les relations entre employeurs et employés de la Fonction publique, a commenté les réalisations du Conseil national mitxe en disposant des questions d'application générale à la Fonction publique mais il a aussi insisté sur les difficultés qui ne manqueraient pas de surgir advenant qu'on fasse droit aux revendications des agents négociateurs à l'effet que soit garanti par la loi le recours à l'intervention exécutoire par des tiers en cas de griefs découlant de l'interprétation ou de l'application des directives du Conseil national mixte. L'auteur du rapport a toutefois intégré au projet de loi certaines dispositions qui permettraient l'accommodement de tout différend que les parties pourraient renvoyer conjointement au Conseil.

\section{LA NÉGOCIATION CONCERTÉE}

Il semble que M. Finkelman soit allé un peu plus loin dans son rapport car il préconise une solution qui inciterait les parties à préférer à la consultation la négociation des questions d'application universelle. Je me reporte plus exactement aux dispositions en matière de négociation concertée. Que ces recommandations soient mises en œuvre et elles permettront à l'employeur ainsi qu'à un, deux ou plusieurs agents négocia- 
teurs de conclure un accord principal visant les conditions d'emploi applicables aux employés de plusieurs unités.

J'ai la certitude que l'importance de cette recommandation ne passera pas inaperçue. À moins de pouvoir adapter les procédures du Conseil national mixte à la solution plus rapide des problèmes et comme moyen de règlement, par des tiers, des différends découlant de l'interprétation ou de l'application de toute directive, tout laisse prévoir que les agents négociateurs chercheront à s'en remettre aux modalités de la négociation concertée.

Même si le Conseil national mixte poursuit sa tâche à un rythme plus lent et comme moyen de régler à l'amiable certaines questions d'intérêt général pour la Fonction publique, la négociation concertée ou les conventions collectives d'application générale n'en sont pas moins appelées à jouer un rôle de tout premier plan dans la Fonction publique.

Disons à titre d'exemple que l'Alliance représente actuellement toutes les unités de négociation de la catégorie du «soutien administratif ». Exception faite de la rémunération, les conditions d'emploi sont pratiquement les mêmes et il est fort peu probable que le Conseil du Trésor ou l'Alliance veuillent apporter d'importantes modifications d'une convention collective à l'autre dans le cas de ces groupes. Il faut toutefois, aux termes du rituel en vigueur, signifier six avis distincts de négocier, produire six séries distinctes de revendications et négocier séparément six fois etc, alors que les conventions collectives sont virtuellement les mêmes. Les ressources du Conseil du Trésor comme celles de l'Alliance sont affectées à ce processus de dédoublement onéreux alors que les employés membres d'unités plus modestes se sentent de plus en plus frustrés car, étant inférieurs en nombre, il leur faut attendre patiemment qu'on ait disposé des groupes numériquement plus importants. Les employés s'estiment davantage frustrés lorsque les négociateurs du Conseil du Trésor semblent vouloir accumuler des «bons points » en prolongeant successivement les conventions collectives négociées. Des conventions collectives d'application générale permettraient d'accélérer les négociations, de pourvoir à des conditions plus uniformes là où il faut maintenir la relativité et cependant, de pourvoir à diverses échelles de rémunération pertinentes aux diverses unités de négociation.

\section{LA COMMISSION DE LA FONCTION PUBLIQUE}

Je prédis que le rôle de la Commission de la Fonction publique du Canada, en matière d'administration des conditions d'emploi et des rela- 
tions du travail, fera l'objet d'une véritable expertise au cours des prochaines années. Les Commissions du Service Civil se sont révélées, au tournant du siècle, comme autant d'organismes extrêmement utiles et comme un moyen nouveau de protéger les fonctionnaires contre les répercussions néfastes du favoritisme politique. Quelques-unes de ces Commissions, et notamment la Commission du Service Civil de Grande-Bretagne, se sont intéressées exclusivement à la réglementation des nominations à la Fonction publique. D'autres, telle la Commission canadienne du Service Civil, dans sa formule originale de 1918, se virent confier des pouvoirs pour toutes ces questions que nous connaissons actuellement sous le titre général $d$ ' «administration du personnel ». La commission canadienne, au fil des ans, allait de plus assumer un rôle quasi-judiciaire dans le cadre des mesures qu'elle prenait elle-même ou que prenaient ceux qui agissaient en son nom.

Au cours des trois premières décennies qui suivirent 1918, les associations d'employés de la Fonction publique - qui ne possédaient aucun droit légal et qui ne pouvaient ordinairement compter que sur un appui limité de la part des fonctionnaires - devaient s'en remettre à la Commission du Service Civil pour obtenir son concours dans leurs démarches visant à protéger les intérêts de leurs membres. Comme le disait si bien le professeur Hodgetts, les rapports entre les associations de fonctionnaires et la Commission du Service Civil étaient forcément ambivalents car ces rapports étaient autant ceux d'adversaires que d'amis et que bien souvent, les associations ne pouvaient apprécier à sa juste valeur le rôle que jouait la Commission ni à quel point, sous le couvert de l'autonomie, elle n'était que l'agent du gouvernement de l'heure.

Trois nouveaux acteurs allaient faire leur entrée en scène à l'avènement de la négociation collective; les employeurs, chargés sans ambiguitté de protéger les intérêts de l'État en sa qualité d'employeur; l'agent négociateur, chargé exclusivement par la loi de représenter les intérêts des fonctionnaires affectés à des unités de négociation dûment constituées; et un organisme de réglementation, la Commission des relations de travail dans la Fonction publique, au rôle quasi-judiciaire et non compromise par des engagements plus ou moins précis envers les employeurs ou le syndicat.

Dans ce nouveau contexte, la Commission de la Fonction publique allait devoir céder à l'employeur nouvellement désigné, le Conseil du Trésor, son contrôle sur la classification, la rémunération, les droits aux 
congés et sur la presque totalité des autres conditions d'emploi à l'exception des nominations à la Fonction publique ou au sein de celle-ci.

La plupart des employeurs du secteur privé sont parvenus à restreindre le rôle des syndicats quant à la réglementation de l'embauchage et de l'avancement. La direction juge tout à fait indispensable à la réalisation des objectifs qu'elle s'est fixés une affectation efficace des titulaires en fonction des exigences précises des postes. En ma qualité de directeur général d'un organisme qui compte 200 employés, il me faut souscrire à ce préalable même si je suis d'avis qu'on devrait tenir compte, jusqu'à un certain point, du degré d'ancienneté lorsqu'il s'agit d'affectations et d'avancement des employés. La Commission de la Fonction publique a, depuis 1967, fait tendre tous ses efforts afin que «les administrateurs administrent », comme elle le préconisait d'ailleurs dans son rapport de 1970. En 1972, plus de 68,000 nominations - soit 79 p. 100 de toutes les nominations à la Fonction publique ou au sein de celle-ci ont été effectuées par les directeurs des programmes en vertu des pouvoirs que leur a délégués la Commission par l'entremise de leurs souschefs. Bien que la Commission ait établi un système de «contrôle» de la dotation en personnel dans les ministères, les agents négociateurs n'en ont pas moins vertement critiqué ce système et, malgré l'expertise spéciale de 1971 et les modifications qui allaient en résulter, l'Alliance de la Fonction publique n'a pas encore la conviction que la Commission a découvert - et encore moins qu'elle ait signalé - des cas de népotisme, de discrimination ou de favoritisme politique.

À la vérité, le rôle de la Commission de la Fonction publique, en sa qualité de gardienne parlementaire du système au mérite, a singulièrement perdu de son efficacité depuis 1967. Aucun des six rapports publiés par la Commission depuis la revision de la Loi ne caractérise les faiblesses $\mathrm{du}$ processus administratif mis au point pour sauvegarder le système au mérite ni ne fait état du moindre cas de discrimination ou de favoritisme politique. Comparez les rapports de l'Auditeur général à ceux de da Commission de la Fonction publique et vous n'en apprécierez que mieux la différence. Bien que la Loi sur l'emploi dans la Fonction publique pourvoit à la révocation des nominations irrégulières ainsi qu'à la révocation des pouvoirs délégués dans le cas des ministères qui n'appliquent pas le système au mérite, je n'ai pu relever, du moins à ma connaissance, et ce, depuis 1967, qu'une seule révocation d'une nomination effectuée aux termes des pouvoirs délégués et pas la moindre révocation des pouvoirs délégués. Si l'on songe à quel point le contrôle effectif des nominations est passé, depuis quelques années, de la Commission aux hauts fonctionnaires 
des ministères, il faudra y voir la preuve du maintien sans compromis du système au mérite par la direction dans la Fonction publique tout entière, ce qui témoignerait éloquemment de l'efficacité des systèmes de surveillance de la Commission, ou encore, la renonciation, par la Commission, aux responsabilités fondamentales que lui avait déléguées le Parlement. S'il faut en croire les témoignages que renferme le rapport du comité de revision de la délégation présidée par Monsieur R. Giroux, en 1972 , il ne subsiste que fort peu de doute quant à la description qui correspond le plus près à la situation.

On comprendra facilement que les fonctionnaires fédéraux comme leurs associations aient hésité, en 1967, à renoncer à la protection que leur offrait la Commission - protection d'ailleurs fort relative - pour s'en remettre uniquement à des rapports de négociation collective ainsi qu'à la nouvelle Commission des relations de travail dans la Fonction publique dont les preuves restaient à faire afin de s'assurer un traitement équitable dans le cadre de leurs rapports avec leur employeur, le gouvernement. Le principe du mérite était largement consacré, comme il l'est encore cela va de soi, dans la Fonction publique tout entière comme il y régnait et comme il y règne encore ce désir presque universel de perpétuer un service où l'aptitude à s'acquitter des fonctions et des responsabilités d'un poste doit primer toute autre considération, tant sur le plan du choix des candidats à nommer à la Fonction publique que sur celui de leur avancement à des degrés supérieurs de responsabilités. C'est un principe auquel souscrivent les employés en général, la direction ainsi que les agents négociateurs. Bien que l'Alliance et d'autres agents négociateurs voudraient qu'un degré d'ancienneté s'inscrive parmi les facteurs dont il faudrait tenir compte à l'avancement des employés, les syndicats de la Fonction publique ne veulent pas d'autre part que les perspectives d'avancement professionnel de leurs membres soient restreintes par des unités de négociation à caractère de ghettos ni par un système d'avancement dont le degré d'ancienneté serait le principal critère.

On peut se demander si, dans ce contexte, l'avenir réserve à la Commission de la Fonction publique un rôle comme garante du système du mérite. L'Alliance de la Fonction publique et, du moins c'est là mon avis, la plupart des autres syndicats de fonctionnaires, ne se sont pas prononcés catégoriquement à ce propos. Nous sommes mécontents des dispositions actuelles qui se résument largement à une parodie et à un trompe-l'œil. Si nous ne pouvons compter sur une Commission vraiment indépendante, les syndicats de la Fonction publique devront, à mon avis, songer à ces deux solutions de rechange : ou bien ils demanderont que 
le rôle de «surveillance » de la Commission soit dévolu à la Commission des relations de travail dans la Fonction publique; ou encore, il leur faudra persuader le Parlement d'intégrer les nominations, les mutations et l'avancement aux questions négociables en vertu de la Loi sur les relations de travail dans la Fonction publique. Il ne saurait être question d'appuyer plus longtemps la forme d'un système au mérite alors que de l'intérieur on en ronge le fond. C'est, à n'en point douter, un secteur où tous les intéressés devront reviser leurs positions dans un avenir très rapproché.

\section{CONCLUSION}

C'est pour mieux comprendre ce que nous réserve l'avenir que je me suis arrêté, de propos délibéré, aux sujets susceptibles d'engendrer les problèmes les plus épineux. Je me suis abstenu d'une foule d'aspects qui tous caractérisent les rapports contractuels comme je me suis abstenu de commenter les réformes positives que M. Finkelman recommande d'apporter au système. Mais peu importe les problèmes ainsi que l'empleur des difficultés à leur apporter des solutions, j'ai la ferme conviction que les syndicats, la direction et les «non-engagés » peuvent tous, par leur apport, instituer un régime plus efficace de relations du travail, propre à perpétuer cette réputation enviable dont jouit, parmi les meilleures au monde, la Fonction publique du Canada.

\section{The Future of Public Service Unionism in Canada}

\section{The First Negotiations}

Before we consider the future, it is necessary to briefly examine the past and the present since any prediction as to what is in store must relate to our present and past experiences.

In the Federal Public Service, we have only had collective bargaining for about six years. We are now in our third round of negotiations. The first was an exhilarating experience with a great deal of mutual good faith, limited expertise on both sides of the bargaining table and a number of very difficult problems. The task of conversion of the former classification system into 72 bargaining units with an occupationally oriented classification system was monumental in scope. We managed to incorporate in those agreements many improvements for our membership, particularly those employees who were previously known as «Prevailing Rate employees ».

* Paper presented at the Canada Industrial Relations Research Institute annual meeting, Toronto, 1974. 
I rather believe the Treasury Board felt that the initial government negotiators had given away the store in the first round. New faces appeared at the bargaining table and improvements were harder to get in the second round; the honeymoon was over yet employees with limited experience in collective bargaining seemed to think that their representatives needed only to ask and the Treasury Board would provide. If their particular desires were not met in the collective agreement, then obviously their negotiators simply had not asked or demanded.

\section{The Present Situation}

The present round of negotiations has been even tougher and at the same time the needs and expectations of employees have increased. Rising expectations result from a number of factors: the principal factor at this time is obviously the rapid inflationary increases in the Consumer Price Index. An employee, particularly one at the lower end of the salary spectrum, is hard hit by inflation. In addition, the T.V. has projected every rich man's life style into every poor man's living room, but rather than being able to acquire that life style, he has difficulty making ends meet. Food prices of basic commodities such as meat, bread, milk, etc. go up weekly, not monthly or yearly as he experienced in the past. Rents continue to go up. His dream of acquiring a home of his own becomes just a pipe dream and his frustrations mount in the process. The timid become tigers. They reject authority and the rules. They are not prepared to play the game as it was planned. They expect immediate, sustained, effective action to meet their demands. What might be considered a great achiement in quieter times is dismissed with an expletive as being little or nothing.

Government employees see employees in private industry turning down $12 \%$ increases in one year, $\$ 2.90$ per hour increases in construction trades and they relate those events to their own collective agreements. As a result, agreements nogotiated at the table using the traditional yardstick of comparability with wages paid to similar employees in the private sector have little chance of ratification. So far this year, five tentative agreements have been rejected by the membership in relation to one agreement prior to this year. One rejected settlement provided over $30 \%$ increase in wages plus some additional fringes over a $251 / 2$ month period. The settlement was negotiated under the pressure of an illegal strike and was the largest negotiated wage increase to date in the Public Service. It was not ratified by the membership and is now awaiting a binding arbitration award.

\section{Causes of Dissatisfaction}

However, it would be unfair and unwise to attribute the present unrest entirely to forces in the immediate environment. The tensions have been building for a decade and are rooted in an increasingly articulated position that wage earners on the lower end of the totem pole are not sharing in Canada's growth and prosperity.

The greatest challenge of collective bargaining in the next few years - in both the public and private sectors - will be how to improve the relative position of workers in this country who have failed to secure a fair share of the fruits of our nation's economic growth and development. 
For the private sector these are the unorganized employees who work at or near the legal minimum wage - and sometimes for substantially less than they are supposed to be guaranteed by law. It is a sad reflection on the otherwise substantial achievements of organized labour in this country that nearly a century after the first employees were organized in Canada, and 35 years after the law provided protection to employees against the use of strong-arm employer methods to restrict the encroachment of the unions, more than two-thirds of Canada's work force remains unorganized.

In the municipalities and provincial institutions a great deal of progress has been made in the past decade, although there is room for improvement in both the form and substance of collective bargaining in many provincial civil services. In the Public Service of Canada, the challenge to the unions is not to increase our coverage, which is within a few percentage points of total, or to secure the right to bargain - a right we secured in 1967 - but to raise the incomes of those who are struggling to survive at the bottom of the heap. There is little doubt that rampant inflation has increased our sensitivity to the plight of our lower-paid members and the problems they face in trying to make ends meet on poverty-line incomes. Perhaps, in our anxiety to assure public servants wages comparable to those paid in the private sector, we have been a party to wage settlements for low wage earners which, although statistically defensible, were morally outrageous. The «percentage increase» syndrome which has permeated the collective bargaining scene in Canada for the past decade, provides for wage increases which purport to maintain relationships between workers at various income levels, but in fact constitutes a statistical camouflage for increasing distortion of the relative affluence of those in high income brackets and the relative deprivation of those in the lower brackets. Percentage wage increases do not reveal the extent of improvement or lack of improvement in purchasing power of those who receive the increase, as anyone whose income is nil can demonstrate. A ten percent increase in a nil. income provides its beneficiary with no increase in purchasing power.

Let us, for example, consider the position of three wage earners during the 60 's whose wages in 1960 were $\$ 3,000, \$ 18,000$ and $\$ 50,000$ respectively and who received annual increases of $5 \%$. Assuming a nil income tax for the low wage earner, an effective tax of $30 \%$ for the middle wage earner and $40 \%$ for the high wage earner, the increase in annual purchasing power at the end of the decade will be $\$ 1,654.00$ for the low wage earner, $\$ 6,947$ for the middle wage earner and $\$ 16,540$ for the high wage earner.

In 1960, the purchasing power of the high wage earner exceeded that of the low wage earner by $\$ 27,000$. In 1969 the purchasing power of the high wage earner exceeded that of the low wage earner by $\$ 41,886.00$.

The aggregate purchasing power of the low wage earner throughout the tenyear period was $\$ 37,734.00$ of the middle wage earner was $\$ 158,482$ and of the high wage earner was $\$ 377,337.00$

Most workers in North American society, including members of the Public Service Alliance, do not believe everyone should receive an identical wage. They believe there should be an appropriate differential in wages and salaries to reflect the different contributions made by different individuals and to provide incentives 
for people to acquire higher skills and education and accept broader and more demanding responsibilities. But I do not believe that they approve of the everwidening arc between those at the lower end of the pay scale and those at the upper end, and the regressive distribution of national income which is the inevitable consequence of our present wage culture.

This ever-widening gap is, I believe, the gravest issue confronting our society today. It says, very simply, that those who eat high on the hog will eat higher as time goes on, and that those who eat low on the hog will soon be surviving if they survive at all - on the tail and the hocks. How long will the mass of low wage earners in this country acquiesce peacefully to this demonstrably discriminatory wage and salary culture? How much can they take without revolt?

\section{The Public Service Alliance's Position}

The Public Service Alliance is attempting to redress the worst effects of this regressive wage cycle in which we have been trapped since we were bargaining in 1967. Our concern for what has been happening is reflected in some of our recent settlements which have modified the standard percentage increase formula with larger increases for the lower grades, either in percentage terms or by coupling percentage increases with flat rate settlements. The recent $\$ 500.00$ across-the-board cost-of-living increase for employees in the Public Service was a modest step in the right direction and one which we shall endeavour to repeat in one form or another. Its impact in percentage terms - even before tax was $10 \%$ for the $\$ 5,000$ wage earner and approximately $1 \%$ for those at the top of the wage spectrum.

However, neither the Treasury Board as the employer nor the unions with which the Board negotiates are free to opt out of the wage culture of the community. Since the Government is committed to a policy of relating salaries paid to their employees to the salaries of taxpayers doing similar work, bargaining agents will have difficulty securing rates of pay for their members in excess of rates paid by good employers in the private sector. Consequently significant change can only be brought about if employees and bargaining agents throughout the country make a substantial effort to find patterns and formulae for wage adjustments that will check the socially immoral wage relationships which have been force-fed by percentage increases for two decades.

When you consider that the average wage in the Federal Public Service today is approximately $\$ 9,700$ and according to the latest statistics of the Treasury Board there are approximately 120,000 employees of the service earning less than $\$ 10,000$ per year, it is understandable that the frustrations of these employees may be manifest in a number of ways.

\section{Difficult Times AHEAD}

Many, of the frustrations of employees will be directed at their unions as well as their employer. While wild cat strikes and demonstrations may occur more 
frequently so will attempts to change bargaining agents or union leadership: not that other agents or leadership will have any simple solutions to the problems.

Union discipline will be difficult to maintain and there is likely to be disrespect for the rules of the union as well as the rules of the employer. This will make the bargaining relationship more difficult since neither union nor management can be sure of the acceptance of any agreement that is made. The employer as a result, is likely to hold back his best offer in order to have something in reserve in the event of non-ratification or a wild-cat strike. The union representative on the other hand will be reluctant to accept and recommend what he normally would consider to be an acceptable offer unless he believes he has solid support for the proposal.

All this, of course, leads to tougher bargaining, less negotiated agreements, more use of dispute settlement machinery and the likelihood of increased disruption and unrest.

The frustrations brought about by inflation and a wage policy that continues to widen the gap between the low wage earner and the high wage earner can provide the greatest test of the capabilities of all those involved in labour relations to maintain a system established to develop and maintain a good collective bargaining relationship between employer and employees with a minimum of stress.

\section{Consultation}

The labour relations system of the public service has been marked by substantial use of consultation between the parties as a means of resolving problems or establishing certain terms and conditions of employment. Generally terms and conditions of employment that were established on a universal basis throughout the service were dealt with in this way. The most noteworthy forum for consultation in the Federal Service is the National Joint Council formed 30 years ago.

There is a marked need for a mechanism that will enable the parties to establish similar working conditions and benefits since employees tend to relate their working conditions to others around them. The decisions reached through the Council have permitted universal application on the same effective date, a circumstance that would be most difficult under the normal bargaining procedures where different collective agreements have different expiry dates. The N.J.C. process also prevents «whipsawing» by either the employer or the unions, a situation that would likely occur if conditions were negotiated separately for all bargaining units.

If the National Joint Council is to continue as a forum for consultation, I believe both unions and management must commit greater resources to it. At the present time, the work of the Council is principally performed by Committees of management and union personnel, all of whom have full-time jobs in their regular 
capacities. Meetings are hard to schedule, decisions are delayed, proposals for change cannot be dealt with expeditiously and employees affected by decision are no longer patient.

Mr. Finkelman, in his report on Employer-employee Relations in the Public Service, has commented on the accomplishments of the National Joint Council in dealing with service-wide issues but in addition draws attention to the difficulties that would ensue in agreeing with the representations made by bargaining agents that final and binding third-party determination be made available by statute for grievances that may arise out of the interpretation or application of directives of the National Joint Council. He has, however, provided a means in the proposed legislation for the resolution of any matter that might be referred jointly to the Board by the parties.

\section{COALITION BARgaining}

It would appear also that Mr. Finkelman, in his report, has gone one step further; a step that may encourage the parties to collective bargaining to move from consultation to negotiation on service-wide matters. I am referring to the provisions for coalition bargaining.

If these recommendations are enacted they will permit the employer and a bargaining agent or two or more bargaining agents to enter into a master agreement containing terms and conditions of employment applicable to employees in more than one unit.

I am sure the significance of this recommendation will not go unnoticed. Unless the procedures of the National Joint Council can be adapted to provide speedier resolution of problems and a means of third-party determination of unresolved issues arising from the interpretation or application of any directive, it seems likely that bargaining agent will seek to usilize the provision for coalition bargaining.

Even if the National Joint Council continues to function as a more leisurely and peaceful means of determining some service-wide issues, there is still a very important place for coalition bargaining or master agreements in the Public Service.

For example, at the present time the Alliance represents all bargaining units in the Administrative Support Category. Terms and conditions of employment, except for salaries, are practically identical and it would be highly unlikely that either the Treasury Board or the Alliance would seek major differences in the collective agreements for these units. The present ritual, however, requires six separate notices to bargain, separate demands, separate negotiations, ect., for virtually the same agreements. The resources of both the Treasury Board and the Alliance are committed to this uneconomic process of duplication while employees in the smaller units become increasingly frustrated because, like the smallest piglet in the litter, they must await their turn at the trough. Additional frustrations materialize when Treasury Board negotiators seek to amass some «Brownie Points 》 by stretching out 
successively negotiated agreements. Master agreements would permit more rapid negotiation, more uniform conditions where relativity should be maintained, yet still provide for variations in rates of pay that would be relevant to the various bargaining units.

\section{The Public Service Commission}

The role of the Public Service Commission of Canada in the administration of terms and conditions of employment and employer-employee relationship will, I predict, come under close scrutiny in the next few years. Civil Service Commissions were an extremely useful invention at the turn of the century, an invention clesigned to insulate civil services from the malign influence of political patronage. Some, like the Civil Service Commission of Great Britain, have been concerned exclusively with the regulation of appointments to the Public Service. Others, like the Canadian Civil Service Commission as constituted in 1918, were assigned authority over all matters which we now identify with the general title «personnel administration». In the course of time the Canadian Commission also assumed a quasi-judicial role in relation to its own actions and those who acted on its behalf.

In the first three decades after 1918, the employee organizations of the Public Services - with no statutory rights and limited support from civil servants generally - looked to the Civil Service Commission for assistance in their efforts to protect the interests of their members. As Professor Hodgetts has pointed out, the relationship of the Civil Service employee organizations to the Civil Service Commission was necessarily ambivalent because it was as much adversary as friend, and in many circumstances the associations had no way of knowing the role that it was playing or to what extent, under the guise of independence, it was simply an agent of the government of the day.

Collective bargaining ushered three new players onto the stage; employers with unequivocal responsibility to protect the interests of government as employer; bargaining agents with legal and exclusive responsibility to represent the interests of public servants in formally designated bargaining units and a regulatory board, the Public Service Staff Relations Board, quasi-judicial in its role and uncompromised by extraneous commitments to employers or the unions. In the process the Commission lost to the newly identified employer - the Treasury Board - control over classification, pay, leave and almost all other terms and conditions of employment except appointment to and from within the Public Service.

Most employers in the private sector have managed to limit the role of the unions in the regulation of appointements and promotions. Management considers that effective matching of job requirements to job incumbents is crucial to the achievement of program objectives. As the chief executive officer of an organization employing 200 employees, I cannot help but agree with that proposition, although I think there should be some recognition of seniority in the assigment and promotion of employees. Since 1967 the Public Service Commission of Canada has bent over backwards to, as it proclaimed in its 1970 report, «Let the managers manage». In 1972 , over 68,000 appointments -79 percent of all appointments rnade to 
or from within the Public Service - were made by program managers under authority delegated to them by the Commission via their deputy heads. While the Commission has established a system to «monitor» staffing actions in Departments, the monitoring system has been severely criticized by the bargaining agents, and despite a special study carried out in 1971 and changes made as a result of that study, the Public Service Alliance is still not satisfied that the Commission is discovering - much less reporting - incidents of nepotism, discrimination of political patronage.

Indeed the role of the Public Service Commission as Parliament's guardian of the merit system has been substantially watered down since 1967. Not one of the six reports it has published since the legislation was revised identifies weaknesses in the administrative processes designed to protect the merit system, or refers to a single incident of discrimination or patronage. Compare the Auditor General's reports with those of the Public Service Commission and you will appreciate the difference. Although the Public Service Employment Act provides for revocation of appointments that have been made improperly, and for withdrawal of delegated authority from departments that do not uphold the merit system, so far as I have been able to determine, since 1967 only one appointment made under delegated authority has been revoked and no delegation has ever been withdrawn. Considering the extent to which the operational control of appointments has shifted in recent years from the Commission to departmental officials, this is either evidence of unflagging support of the merit principle by management throughout the service, a clear reflection of the efficiency of the Commission's surveillance systems, or a cop-out by the Commission from the fundamental responsibility vested in it by Parliament. If the evidence in the report of the delegation review team headed by Mr. R. Giroux in 1972 is to be believed, there is little doubt about which description provides the most accurate assessment.

In 1967 civil service employees and their organizations were understandably reluctant to abandon the protection of the Commission - uncertain as it was - and to rely entirely on the collective bargaining relationship and the new and untried Staff Relations Board to assure them a fair shake in their relationships with their employer - the Government. There was, and of course there still is, a strong commitment to the merit principle throughout the Public Service and an almost universal desire to preserve a service where fitness to perform the duties and responsibilities of a job is the primary consideration, both in the selection of persons for appointment to the service and their promotion to higher levels of responsibility. This position is shared by employees generally, by management, and by the bargaining agents. While the Alliance and other bargaining agents would like to see length of services as one of the considerations to be taken into account in considering employees for promotion, Public Service unions do not want the career opportunities of their members circumscribed by narrow bargaining unit ghettos, nor a promotion system in which seniority is the only or the primary criterion for advancement.

In this context, is there a continuing role for the Public Service Commission as guarantor of the merit system ? The Public Service Alliance, and I think most of the other Public Service unions, have no firm position in this regard. We are 
dissatisfied with the present arrangements, which to a very considerable degree, are a farce and a fraud. If we cannot have a Commission that is truly independent, then I think the Public Service unions will have two alternatives : either they will have to seek the transfer of the Commission's «watchdog» role to the Public Service Staff Relations Board, or they will have to attempt to persuade Parliament to make appointments, transfers and promotions bargainable matters under the Public Service Staff Relations Act. We cannot go on supporting the form of a merit system where the substance is being whittled away from within. Clearly, this is an area in which all concerned will have to re-examine their positions in the very near future.

\section{CONCLUSION}

In looking at the future I have purposely focused on those issues that are likely to pose some of the more serious problems. I have not dealt with many subjects that are common to all bargaining relationships nor the positive changes in the system that are being advocated by Mr. Finkelman. Regardless of the issues and the degree of difficulty in attaining solutions, I am convinced that unions, management and the «neutrals》 can all contribute to a better system of labour relations that will maintain the enviable reputation of Canada's Public Service as being one of the best in the world.

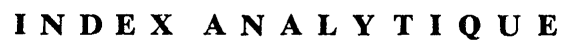
C U M U L A T I V E I N D E X

RELATIONS INDUSTRIELLES INDUSTRIAL RELATIONS

Volume 1 - Volume 25

(1945-1970)

\section{Rapport des - Proceedings of CONGRÈS DES RELATIONS INDUSTRIELLES}

(1946-1970)

Avant-propos - Forewords; A- Index alphabétique - Subject index; B-1 Index méthodique - Broad Subject Headings Index; Liste des descripteurs génériques - List of generic keywords; B-2 Index méthodique des titres - Broad Subject Heading Index of Titles; $C$ - Index méthodique des ouvrages recensés - Broad Authors of Book Reviews; E- Auteurs des articles et des recensions - Authors of the Articles and Book Reviews; F- Références bibliographiques - Bibliographical References.

Un volume $6 \times 9$, relié, 360 pages

prix : $\$ 10.00$

En vente chez votre libraire ou chez l'éditeur

LES PRESSES DE L'UNIVERSITÉ LAVAL

c.p. 2447, Québec 2, (Qué.) 